\title{
Dermières études sur les sécheresses à l'échelle continentale
}

PAR

\author{
J.A. Rodier \\ Chef du Service Hydrologique de loRstom \\ Conseiller Scientifique à Electricité de France
}

Les études des sécheresses sur de vastes territoires présentent toutes une série de difficultés.

Il convient d'abord de définir ce qu'est la sécheresse et c'est là que l'on bute sur les premiers obstacles : c'est que la notion de sécheresse n'est pas la même pour les différents utilisateurs de l'eau. La seule définition générale sur laquelle on arriverait peut-être à un accord est celle que donna il y a un certain temps L. Serra. La sécheresse est relative; c'est une diminution anormale des apports en eau à certaines époques de l'année. Elle ne peut donc pas être confondue avec l'aridité qui, pour certains régimes, correspond à des conditions normales.

Ce déficit en eau peut affecter les précipitations, ou l'écoulement ou les deux à la fois. Elle peut concerner l'ensemble de l'année, la saison pluvieuse, ou simplement un moment critique pour la végétation ; dans ce dernier cas, les agriculteurs peuvent considérer qu'il y a eu une période de sécheresse, bien que les apports totaux des rivières aient été tout à fait normaux. Inversement, il n'est pas rare d'observer des années pour lesquelles les précipitations présentent un total relativement déficitaire, alors que la distribution dans le temps de ces précipitations a été excellente pour les cultures.

L'écoulement annuel étant fonction, non seulement du montant de la hauteur de précipitations annuelle, mais aussi de sa distribution temporelle, il peut y avoir déficit pour la hauteur de précipitations annuelle sans qu'il y ait déficit pour l'écoulement anmuel. Les conditions optimales pour le remplissage des nappes sont loin de correspondre aux conditions optimales pour l'écoulement superficiel ou l'agriculture.

Reconsidérant l'importance de l'époque du déficit par rapport au cycle de végétation, on arrive à des distinctions plus subtiles. Dans les zones arides, pour ce que l'on appelle les pâturages, il n'est pas nécessaire que la majorité des graminées puissent atteindre le stade de la fructification, alors qu'il n'en est pas de même pour les cultures sèches dans les mêmes zones. Une même année peut donc correspondre à des conditions acceptables pour l'élevage et catastrophiques pour la culture du mil.

Cependant, dans les cas extrêmes, la sécheresse affecte tolis les usages de l'eau et il y a sécheresse pour tout le monde.

Avant de procéder à une étude générale, il convient donc d'adopter une définition plus étroite de la sécheresse : sécheresse agricole ou déficit global de l'écoulement annuel cher aux hydrologues d'Electricité de France qui utilisaient largement la notion d'hydraulicité annuelle. L'auteur n'a jamais réussi à faire comprendre à certains hydrologues étrangers ce concept d'hydraulicité

On peut se baser aussi sur le déficit de la hauteur annuelle de précipitations. Mais à chaque fois il convient de préciser de quelle sécheresse l'on parle.

Ces difficultés dans la définition expliquent en partie pourquoi, au cours de la Décennie Hydrologique Internationale, si peu de choses aient été faites dans ce domaine de l'hydrologie : il y a eu de nombreux "dialogues de sourds" qui, bien entendu, se sont ierminés sans résultat.

$\mathrm{Si}$ on considère un critère simple de sécheresse, par exemple l'écoulement total annuel, on se heurte à un autre genre de difficultés : on peut dire que, même dans les régions à régime simple, la sécheresse n'est homogène ni dans l'espace ni dans le temps. L. Serra avait étudié autrefois les périodes de sécheresse bien connues en Europe Occidentale en ne considérant que la notion d'hydraulicité annuelle. Il avait constaté que la super- 
ficie déficitaire couvrirait la majeure partie de la zone étudiée mais que des régions importantes présenteraient une hydraulicité excédentaire.

Lors de la dernière sécheresse au Sahel, on a constaté. d'après le même critère d'écoulement annuel, que malgré l'énorme étendue affectée, du Brésil jusqu'à l'Australie, certaines régions avaient été privilégiées pour une ou deux années de la période de sécheresse et présentaient même parfois un léger excédent. En outre, de 1971 à 1973. suivant les régions, le paroxysme de sécheresse n'avait pas lieu la même année : il y avait donc non seulement hétérogénéité dans l'espace mais aussi dans le temps. Ceci complique singulièrement les études de synthèse.

Enfin, le dernier ensemble de difficultés réside dans létude des causes mêmes de la sécheresse qui nécessite des analyses à l'échelle mondiale des phénomènes qui sont à la base des déplacements des masses d'air.

Comme on l'a indiqué plus haut, ces problèmes attiraient relativement peu les hydrologues lorsqu'en 1971, et surtout en 1972, ce fléau ravagea le Sahel Africain et d'autres parties des zones tropicales. La sécheresse prit ensuite une place prépondérante dans les préoccupations de tous les spécialistes qui s'intéressaient aux problèmes de l'eau. Ceci se traduisit par un très grand nombre de visites d'experts, des études également nombreuses. Malheureusement, les crédits disponibles pour les mesures ont été infiniment plus modestes que pour les voyages d'experts, pour plusieurs raisons : lorsqu'une période de sécheresse commence on ne sait pas, bien entendu, si elle sera sévère ou non; en plus, le travail ingrat d'exploitation du réseau pluviométrique ou hydrométrique est souvent négligé pour des analyses hydrologiques plus brillantes. Les conséquences ont été les suivantes pour l'Afrique sahélienne : la qualité des données pluviométriques correspondant aux réseaux les plus pauvres est médiocre : dans bien des cas 15 à $20 \%$ des postes seulement ont fourni des données sûres.

Pour les données hydrométriques, la situation est nettement meilleure sans être très bonne. Les Services de l'Hydraulique du Sénégal et du Mali et le Service du Génie Rural du Niger, aidés par l'ORSTOM, ont réussi dès le début à obtenir des relevés sûrs pour les stations principales des grands fleuves et surtout à mesurer avec précision les valeurs minimales absolues des débits, qui étaient très en dessous de tous les débits mesurés jusqu'ici. En outre, pour les réseaux de stations de jaugeages organisés sur les cours d'eau de moyenne importance du Sahel (Niger, Haute-Volta), les relevés donnent satisfaction. Une subvention de la DGRST à l'ORSTOM a grandement facilité l'exécution des mesures pendant la demière partie de la sécheresse. Mais mahneureusement, pour les cours d'eau sahéliens et subdésertiques non contrôlés par les réseaux, aucune enquête a posteriori n'a pu être faite faute de temps et d'argent et pourtant il aurait été facile de faire, pour chacune des années 1971 - 1972 - 1973, l'inventaire des cours d'eau n'ayant présenté aucun écoulement annuel il est trop tard maintenant. Sur le plan pratique, ces informations auraient été essentielles.

D'après les quelques informations à notre disposition. les données de base ne sont ni meilleures ni plus sûres dans les autres régions tropicales qui ont été affectées par la sécheresse ; on peut malgré tout se faire une idée de la sévérité de celle-ci. Mais les hydrologues qui ont travaillé sur le terrain n'ont pu s'empêcher de faire le rapprochement entre l'indigence des moyens dont ils disposaient et l'extraordinaire agitation dont ils ont ćté témoins : missions, réunions, création de diverses Commissions, le tout faisant sérieusement double emploi, personne ne voulant donner l'impression d'être resté inactif. Nous tenons cependant à préciser que des choses utiles ont été faites, généralement par les organismes qui ont fait le moins de bruit, et que les envois de vivres auraient été nettement suffisants si la majeure partie était parvenue aux intéressés.

On peut dire que l'ensemble des études à effectuer sur un tel sujet comporte trois volets

1) le bilan de la situation : en ce qui concerne l'Hydrologie, étude du déficit et de sa fréquence

2) l'analyse des causes profondes;

3) la recherche des moyens pratiques propres à remédier à de telles catastrophes.

Dans le présent exposé, on s'attachera surtout à l'examen des deux premiers volets.

Dans un premier temps, les études des données pluviométriques et hydrologiques ont été faites par l'ASECNA et l'ORSTOM. Puis assez vite il s'est avéré nécessaire de travailler avec les organisations internationales pour deux raisons : d'abord parce que, comme la sécheresse de 1958 dans les régions équatoriales, celle de 1971-1973 (ou 1965-1973) affectait la majeure partie des régions tropicales du monde, y compris le Nord-Est du Brésil, les Antilles, les Indes, l'Australie etc. et il importait d'élargir le champ des études ; ensuite, pour éviter de refaire plusieurs fois les mêmes études, il valait mieux qu'il y ait un peu de coordination au milieu des rapports successifs ou simultanés sur ce sujet.

Un bon rapport d'ensemble avait été rédigé par E.G. Davy, expert de l'OMM ; un rapport ne traitant que des aspects hydrologiques et pluviométriques a été mis au point fin 1973 début 1974 conjointement par l'OMM, plus particulièrement par son rapporteur sur la sécheresse continentale, et par l'Association Internationale des Sciences Hydrologiques avec un large concours de l'ORSTOM. Le rapport, assez complet pour l'Afrique, était très pauvre pour les autres continents. Peu après, une autre série d'études d'ensemble était entreprise en France, sous l'égide de la Coopération ou de la DGRST. Elles étaient plus ou moins liées à la recherche des moyens de lutte contre les conséquences des sécheresses : étude générale des ressources en eaux souterraines du BRGM, étude générale des modèles d'écoulement en région sahélienne (ORSTOM), étude de l'écoulement annuel dans le Sahel tropical africain (ORSTOM), recherche d'une "stratégie antisécheresse" (SCET-Intemational avec le concours de l'ORSTOM). Les modalités de l'écoulement dans le Sahel, y compris pendant les périodes de sécheresse, étaient approfondies et mises sous une forme pratique pour guider l'élaboration des projets d'aménagement. Des études individuelles étaient effectuées sur les causes de la sécheresse et, aux EtatsUnis, de grands organismes de recherches tentaient d'utiliser tout l'arsenal des modèles météorologiques.

Mais le Sahel n'avait pas été le seul frappé par la sécheresse. L'URSS a eu des déficits sévères et même la France a connu, à la même époque, des périodes de déficit sérieux. C'est pourquoi, au Comité exécutif de l'Union Géodésique 
et Géophysique Internationale, l'Association Internationale des Sciences Hydrologiques avait demandé qu'un Symposium soit organisé à l'Assemblée Générale de l'UGGI sur les aspects hydrologiques et météorologiques des sécheresses continentales. On espérait ainsi recevoir un bon nombre de communications sur la sécheresse dans les zones tempérées et sur les causes de sécheresses. C'était un Colloque conjoint AISH et AIMPA, sous la direction d'AIMPA. Nous avons été assez déçus : 16 auteurs s'étaient inscrits; sur les 16 Communications, 10 traitaient le sujet et encore, sur ces 10 Communications, certaines n'ont pas été présentées. Très peu de chose a été dit sur la sécheresse dans les zones tempérées. Par contre, les rapports ont été un peu plus nombreux sur les causes de ce phénomène : l'un des plus concrets a été présenté par L. Dorize qui montre clairement la liaison étroite entre la pénétration de la mousson sur le Continent africain et les fortes expulsions cycloniques au large de l'Antarctique dans la mer de Wedell. Une cyclogénèse déficiente sur l'Antarctique Sud, une activité affaiblie du front polaire, conduisent à une pénétration faible de la mousson et à la sécheresse dans la zone tropicale Nord et surtout sahélienne. Ces considérations permettent de prévoir des possibilités de prévisions à moyen terme.

Deux modèles sur la circulation dans le désert ont été présentés : l'un de J.G. Chamey (USA) liant l'augmentation de l'albédo dans les zones marginales du désert à la création et à la persistance de la sécheresse, cette variation étant due au surpâturage. Pour qui connaît la nature des "verts pâturages" du Sahel, cette hypothèse basée sur une vaste étude sur modèle à l'échelle de l'Afrique parait peu convainquante, d'autant plus qu'après la première année de sécheresse, presque tout le bétail étant mort, il n'était plus question de surpâturage. Une étude analogue sur modèle a été faite pour le Negev.

Un exposé russe très complet sur les déplacements, d'une latitude à l'autre, des systèmes de circulation, sur le régime thermique des océans, y compris les mers arctiques, et enfin sur les fluctuations de l'activité solaire et leurs rapports avec la sécheresse, n'a malheureusement pas été présenté.

Deux exposés traitaient de l'influence du rôle de la température de surface des océans sur la genèse des systèmes de circulation des masses d'air conduisant aux sécheresses, lesquelles tendent à s'entretenir elles-mêmes (phénomène de persistance étudié pour le Sahel par Y. Brunet-Moret et M. Roche en 1975). Deux exemples concernent le Sud des Etats-Unis en 1952-1954 et la partie européenne de l'URSS en 1972.

Un rapport soviétique exposait l'ensemble des sécheresses depuis le $9^{\mathrm{e}}$ siècle, avec des pseudo-cycles de 7 à 14 ans liés aux phases de l'activité solaire ou géomạnétique.

Un rapport mexicain présentait les liaisons entre les fluctuations de la circulation de l'atmosphère dans l'hémisphère Nord et la sécheresse. Enfin, un modèle stochastique par point, pour les sécheresses continentales à deux ni- veaux, a été mis au point aux Etats-Unis, le premier niveau représente les mécanismes générateurs des précipitations, le second présente les précipitations elles-mèmes, ce modèle donne de bons résultats dans le Nord des Etats-Unis. Il est bon de noter que, dans le Colloque sur les changements climatiques, on trouve quelques éléments utiles pour le problème des sécheresses : par exemple, un exposé de A.L. Berger sur les transports d'énergie vers les pôles depuis les 100000 dernières années jusqu'aux 100000 prochaines années ; or les hautes latitudes jouent un rôle essentiel dans la circulation de l'atmosphère qui conditionne les sécheresses.

Enfin, une Communication avait été rédigée par $\mathrm{J}$. Sircoulon sur les aspects hydrologiques de la sécheresse récente en Afrique. Il en présentera tout à l'heure une version mise à jour et beaucoup plus complète. Les exposés les plus intéressants du Colloque de Grenoble seront publiés probablement dans le Bulletin de l'AISH.

On a un peu parlé, au cours du même Symposium, des moyens de lutte contre la sécheresse : possibilités de prévision, amélioration des méthodes de recherche des eaux souterraines, utilisation des eaux légèrement salées, multiplication des points d'eau : forages ou très petits réservoirs de façon à limiter la surface de paturage desservie par chaque point d'eau et à diminuer les risques de surpâturage. On a dit, en exagérant un peu, qu'en 1972 il $y$ avait de l'eau là où il n'y avait pas de pâturages et des points d'eau à sec là où les bêtes auraient pu trouver à se nourrir. Une étude d'ensemble des nappes souterraines principales a été entreprise par le BRGM tandis que l'ORSTOM s'efforçait de fournir des normes de calculs hydrologiques pour les petits réservoirs.

Signalons un rapport récent publié par l'Académie des Sciences d'Outre-mer "La sécheresse en zone sahélienne", ouvrage mis au point par un groupe pluridisciplinaire de spécialistes dont une des principales conclusions est la suivante : "Si un aménagement systématique des points d'eau dans la zone d'élevage est nécessaire, de même que l'utilisation rationnelle des cours d'eau nés dans le Sahel pour l'irrigation et l'élevage est également nécessaire, les ressources qui en résulteront seront tout à fait insuffisantes pour la population des Etats du Sahel, telle qu'elle sera dans vingt ans, surtout en période de sécheresse. Le seul moyen d'y parvenir est l'aménagement intégral des grands fleuves : Sénégal, Niger (non seulement le Delta mais le Niger Moyen depuis l'amont de Niamey jusqu'à la frontière du Nigeria, le Chari, le Logone et le Nil. Bien entendu, ceci suppose irrigation et pompage. En 1972, les quantités d'eau débitées par le Logone étaient largement suffisantes, mais la cote était telle que très peu de rizières ont pu être irriguées.

Ces aménagements sont coûteux, mais, depuis les sécheresses de 1913 et 1972 et les crues de 1955, 1961 et 1967, on dispose de suffisamment de séries de données hydrologiques de longue durée pour pouvoir effectuer, à partir de ces bases, des études hydrologiques sérieuses pour l'étude de ces projets. 


\section{Discussion}

M. le Président remercie M. ROCHE d'avoir remplacé si efficacement M. RODIER pourl'exposé de sa communication et poursuit :

Ayant eu loccasion, il y a deux ans, de faire une mission au Niger, j'ai pu apprécier l'importance et l'intérêt des relevés hydro. logiques effectués dans cette région par l'ORSTOM. J'était arrivé à la conclusion qui rejoint celle de M. RODIER, à savoir que pour permettre le développement convenable des irrigations dans la vallée du Niger, notamment en République du Niger, il est indispensable de créer sur ce fleuve des retenues relativement importantes. Parmi les projets envisagés, il faut citer le barrage de Kandadji à l'amont de Niamey qui a les plus grandes chances d'être réalisé ; la retenue de ce barrage s'étendra d'ailleurs sur une partie du territoire de la République du Mali.

M. TIXERONT donne un point de vue tiré de sa longue expérience au cours d'une intéressante intervention qu'il a résumée comme suit :

Il y cut entre les années 1940 et 1950 une sécheresse extraordinaire qui a affecté tout le bassin méditerranéen. Y-a-t-il une étude d'ensemble de cet événement?

Il est possibie, en effet, d'en tirer un certain nombre d'enseignements : une catastrophe de cette nature vaut une période de récurrence de l'ordre d'un siècle en Italie ; en Tunisie, le Sahara avait progressé d'une centaine de kilomètres vers le Nord. Les remèdes étaient naturellement la mise en ceuvre des moyens de stockage pour lesquels, comme le fait remarquer M. RODIER, le stockage de l'eau d'irrigation dans l'aménagement des grands fleuves est le plus efficace.

Il y a lieu de noter que les populations d'une région sont diversement affectées; les gens qui risquent de mourir de faim sont les habitants des zones marginales. . C'est-à-dire, sans doute, dans le Sahel soudanais, les zones du Nord. A supposer qu'il faille faire venir des vivres de l'extérieur, les quantités sont limitées et il n'en est besoin qu'en de rares années ; il est alors essentiel de disposer d'un bon système de transport et de distribution, tels que ceux dont on disposait en Tunisie.

M. RODIER a critiqué l'abus qu'on fait de la notion de surpâturage. Je suis bien d'accord à ce sujet. En réalité, la charge de bétail s'équilibre forcément avec les possibilités de production de lherbe. Il y a établissement d'un "climax". Ce n'est pas foncièrement différent de l'équilibre entre faune sauvage et végétation. Dans les deux cas, bien entendu, cet équilibre est fluctuant.

Ceci m'amène à ajouter une remarque à celle que j'ai faite ci-dessus après l'exposé de M. RODIER.

Les stocks, de quelque nature qu'ils soient, peuvent être considérés de deux façons : stocks à long terme compensant la déficience des pluies, stocks à court terme donnant le temps de se retoumer si survient un événement imprévu donnant, par exemple 3 mois de délais au cours desquels on peut préparer des mesures telles que liquidation, dans de bonnes conditions, du bétail excédentaire, acheminement de secours d'urgence, etc".

Répondant à la question posée par M. TIXERONT au début de son exposé, M. ROCHE indique qu'il n'existe pas, à sa connaissance, d'étude de la sécheresse 1940-1950 pour l'ensemble du Bassin Méditerranéen. Il signale que, pour le Sahel, à l'occasion de l'analyse de la dernière sécheresse, on a repris l'étude de la sécheresse de 1940-1950 et de celle qui, en 1913, avait amené le lac Tchad à un niveau minimal comparable à celui atteint ces dernières années.

\section{Abstract \\ Recent studies of droughts on a continental scale}

It is extremely difficult to make a synthesis of drought conditions on a regional scale. Drought must first be defined in accurate terms, but unfortunately, such definitions vary among different water consumers. Farmers and water power engineers have very different ideas on the subject, except in cases of very acute water shortage. L Serra defines drought as an abnormal decrease in water supplies at certain times of the year, or in specific cases during the whole year. Precise definition of the type of drought is essential before undertaking a general study of the subject. In addition, droughts lack homogeneity in both time and space, even when considered on a small scale.

A considerable number of studies were made of the recent drought in tropical Africa (which includes the Sahel region), which were based on frequently unreliable rainfall records and river or lake gauging data which sometimes left to be desired. Nevertheless, this information provided a reasonably clear picture of the calamity which struck not only a major part of Africa, but also Brazil, Australia and the Indian sub-continent. A first report on the subject was produced by E.G. Davy, a World Meteorological Organization expert, followed in 1974 by a joint WMO/IAHS report on the hydrological aspects of the drought.
A series of studies were undertaken in France by B.R.G.M., S.C.E.T. Internationale and O.R.S.T.O.M, which were financed by the French Directorate-General for Scientific and Technical Research (D.G.R.S.T.) and the Ministry of Cooperation. All these studies were intended to provide practical, if possible quantitative data for use in planning counter-measures against the after-effects of the drought.

Comprehensive research programmes have been undertaken in Great Britain and the United States on the subject of the Sahel drought, and in France, the hydrological and meteorological aspects of droughts on a continental scale were discussed at a Symposium organized by various international and local bodies in Grenoble in September 1975. The results of this meeting were sowewhat disappointing, as only few reports related to temperate regions or made reference to the causes of drought. From the purely scientific angle, therefore, much remains to be done before the factors involved can be really understood. On the practical side, a realistic report by the "Académie des Sciences d'Outre-Mer" outlining a practical water management policy for the Sahel and neighbouring regions of West Africa will no doubt be of interest. 